\title{
Identification of Non-convex Blocks on the Edges of a Tunnel
}

\author{
Jianyong Lia, Guangxiang Yuan, Yang Zhao, Jinhu Hu \& Zhiquan Huang \\ Department of Underground Space Engineering, School of Resources and Environment, North \\ China University of Water Resources and Electric Power, Zhengzhou, 450045, China
}

Keywords: tunnels, edges, non-convex blocks, block theory.

\begin{abstract}
In the tunnel engineering, concave edges can be formed by the intersections of roof and wall, floor and wall, wall and wall. If joints or fractures inside the rock mass cross the roof and wall at the same time, instable non-convex blocks can be formed possibly. However, the theorem of finiteness on the convex blocks in the classical block theory does not apply to the non-convex blocks. Combined with practical engineering example, all the simple convex blocks near the edge of a tunnel can be analysed by enumeration, and all the finite or removable convex blocks will be searched out. Thereafter, all the possible finite or removable non-convex blocks will be identified according to the principle that the codes of the joints keep the same all the time. The combination of convex blocks which are overlapped with each other can also be optimized further. Therefore, the non-convex blocks will be represented with several convex blocks which are connected only at the cutting planes. The mode and direction of sliding can be analysed with the stereographic projection and the visualized block sphere. Finally, the identification of non-convex block on the edges of tunnels will be realized. It not only have an active theoretical significance for extending the range of application of block theory, but also have the important reference values for protecting the safety of tunnels engineering.
\end{abstract}

\section{Introduction}

There are a lot of tunnel engineering in highway, railway, water conservancy and other industries. The stability of surrounding rock such as roof fall and collapse is an outstanding problem of influencing the safety of a tunnel. Therefore, it is an important work for designers to identify the instable blocks from the surroundings of the tunnel, and is also one of the core problems in rock mechanics.

The essence of studying the stability of the surroundings in the tunnel is to study the mechanism physical and mechanical changes of rocks, and the distribution of stresses after excavation of a tunnel in the rock mass. The present methods of studying the stability of surrounding rocks are: the calculation of mathematics and mechanics, the analysis of mechanism of deformation and failure of rocks, the analysis of geological structures, the classification of stability of the surrounding rocks and the simulation experiment method. These methods are mostly to start from the description and simulation of boundary conditions and constitutive equations, and to reveal the processes of deformations and failures of rocks as their purposes. The main characteristics of these above methods are the analysis of stress and strain. The other type of stability analysis is the theory of limit equilibrium. Establishing the equilibrium equations of the critical state between the deformation and the failure are the main means. The critical sliding forces and the related resistances are the two contrary aspects. It is the peculiarity of limit equilibrium analysis to obtain the factor of safety [1-3].

In general, majority of blocks in the walls, roof or floor of a tunnel are convex, and have been studied maturely in the classical block theory [4-8]. Concave edges, however, can be formed with the intersection of roof and wall, floor and wall, wall and wall. If the joints or fractures inside the rock mass cross the roof and walls at the same time, instable non-convex blocks may be formed probably, which is not applied to by the theorem of finiteness on the convex blocks in the classical block theory [9-10]. According to the analysis of finiteness and removability on the system of convex blocks, the purpose to identify the non-convex block in the edge of a tunnel can be realized 
with the theory of united blocks.

\section{Combination of the Half-spaces in the Tunnel Edges}

\section{Interpolation function}

The excavation surfaces of a tunnel and the joints or fractures inside the rock mass can be abstracted to be planes in space.

$$
A x+B y+C z+D=0
$$

Space can be cut into two half-spaces by a spatial plane, upper half-space and lower half-space, or left half-space and right half-space.

$$
A x+B y+C z+D>0 \text { or } A x+B y+C z+D<0
$$

A convex block can be analyzed by the intersection operation of its surfaces half-spaces; a concave intersection of two half-spaces, however, should be operated with union for a non-convex one. Concave edges can be formed by the intersection of wall and wall, roof and wall, floor and wall inside the tunnel, as shown in Fig. 1.

\section{Fast non-dominated sorting.}

Concave edges can be formed by the intersection between wall and wall inside the tunnel, so convex edges can. In the case of concave edges, as shown in Fig. 2, four vertical concave edges are formed with four vertical walls intersected with each other. According to the stereographic projection, excavation pyramids in the edges are the unions of solid half-spaces of walls, and are the complements of space pyramid. That is to say, the space pyramids in the edges are the intersections of empty half-spaces of walls.

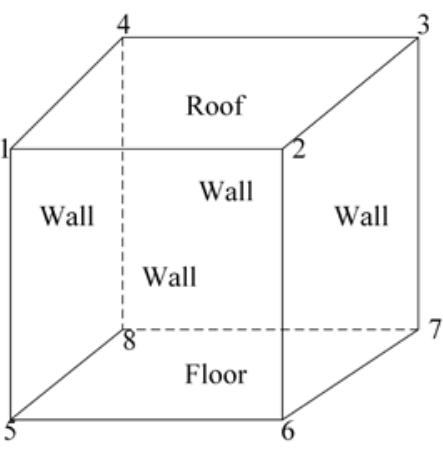

Fig.1: The floor, roof and walls of a tunnel

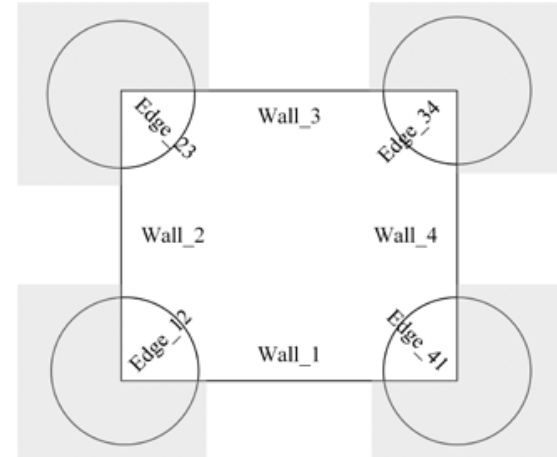

Fig.2: The excavation pyramid between wall and wall

\section{Define the crowding distance.}

Concave edges are formed by the intersection of horizontal roof or floor and vertical walls inside the tunnel, as shown in Fig. 3 and Fig. 4. It can be seen that the areas of space pyramids in Fig. 4 are smaller obviously than the ones in Fig. 3. This shows that the probability of key blocks formed by the wall and the floor is much lower than the one by the wall and the roof.

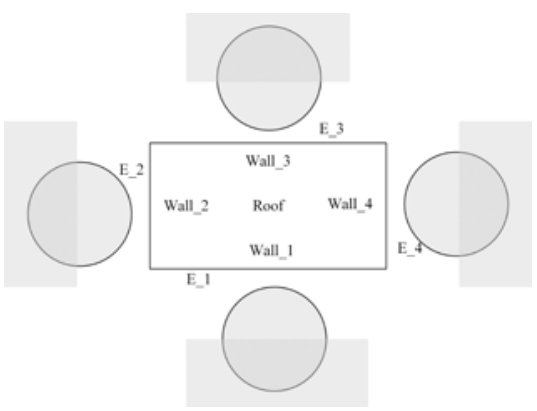

Fig.3: The excavation pyramid between roof and wall

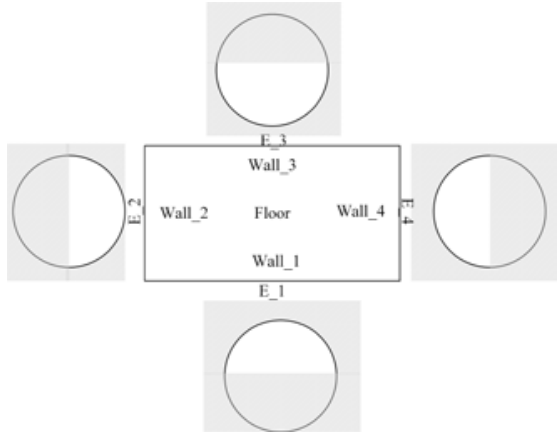

Fig.4: The excavation pyramid between floor and wall 


\section{Identification of Non-convex Blocks on the Tunnel Edges}

\section{Finiteness and Removability of United Block}

In the classical block theory, the theorem of finiteness that whether a block is finite is judged by the block pyramid is aimed at convex blocks, and does not apply to the non-convex blocks. As the non-convex block is concerned, only necessary condition is proved to be right. That is to say, if a non-convex block is finite, its block pyramid is empty, and not vice versa.

Since it is not feasible that whether a block is finite is judged by pyramid, the characteristics of the non-convex block can be studied from the view of united block. Two propositions about the relationships between united block and its sub blocks have been proved: the identification criteria of finiteness and removability. In accordance with the two criteria, the non-convex block in the edges of a tunnel can be analyzed and identified.

\section{Complete Combination of Non-convex Block}

From the experience of practical engineering, the number of joints are no more than five groups in site. Along with the limited number of free planes, all the possible convex blocks which may be sub blocks of a non-convex block can be analysed with an enumeration method.

For a block system that consists of $M$ sets of joints and $N$ free planes, all the joints are intersected convexly, and the free planes contain concave combinations. The space can be cut into $4^{(M+N)}$ types of combinations theoretically. In the practical engineering of rock tunnels, only two codes 0 and 1 are considered for the joints. For the free planes with concave combinations, only three codes 0,1 and 2 are considered. The values of $M$ and $N$ are usually no more than 5 . Then the number of codes combinations to be considered is: $2^{M} \times 3^{N}-2^{M}=2^{M}\left(3^{N}-1\right)$. It can be seen that the case of joint blocks without free planes should be excluded.

The non-convex block is to be viewed as a combination of several convex blocks. Firstly all the possible finite convex blocks and removable ones are searched out by enumeration according to the theorem of finiteness and removability in the classical block theory. After that, the convex blocks can be combined by the rules that the codes of the joints should be the same. Finally, all the possible finite non-convex blocks and the removable ones may be obtained in the system of the spatial planes.

\section{Optimized Combination of Non-convex Block}

According to the analysis of enumeration, while a non-convex block is represented by a group of convex blocks, these convex blocks are overlapped with each other. An optimized method is to cut the rock along with any free plane without the other free planes at that time. Then a convex block can be determined from the complete set of convex blocks. Furthermore, the code of the other halfspace of the cutting plane on the remaining block can be deduced according to the code of the convex block obtained before.

The volume of a non-convex block is the algebraic sum of the volumes of all the convex blocks, so its surface area is, except that it should be subtracted two times of areas of the cutting planes.

\section{Case Validation}

\section{Analysis of Convex Blocks in the System of Spatial Planes.}

A system of spatial planes consist of three joints ${ }^{P_{1}}, P_{2}, P_{3}$ and two free planes ${ }_{4}, P_{5}$ in an edge of a tunnel. The solid half-spaces of ${ }^{P_{4}}$ and ${ }^{P_{5}}$ are intersected concavely. The location and direction of the spatial planes are shown in table 1.

Table 1: The system of spatial planes in the edge of the tunnel

\begin{tabular}{lllllll}
\hline$P_{i}$ & $\alpha_{i}$ & $\beta_{i}$ & $X_{i}$ & $Y_{i}$ & $Z_{i}$ & $\varphi_{i}$ \\
\hline$P_{1}$ & $90^{\circ}$ & $0^{\circ}$ & 1.0 & 0.0 & 1.0 & $30^{\circ}$ \\
$P_{2}$ & $45^{\circ}$ & $135^{\circ}$ & 0.0 & 0.0 & 1.0 & $25^{\circ}$ \\
$P_{3}$ & $90^{\circ}$ & $270^{\circ}$ & 0.0 & -1.0 & 1.0 & $35^{\circ}$ \\
$P_{4}$ & $30^{\circ}$ & $0^{\circ}$ & 0.0 & 0.0 & 0.0 & -- \\
$P_{5}$ & $30^{\circ}$ & $270^{\circ}$ & 0.0 & 0.0 & 0.0 & -- \\
\hline
\end{tabular}


The number of block pyramids of convex blocks are: $2^{3}\left(3^{2}-1\right)=64$. According to the theorem of finiteness in the classical block theory, fourteen finite convex blocks can be obtained: 00001, 00010, 00011, 00012, 00021, 00110, 01110, 10001, 11001, 11100, 11101, 11102, 11110, 11120.

All the possible non-convex blocks are as shown in table 2, according to the principles that the codes of joint pyramid keep the same all the time, and the identification criteria of finiteness of nonconvex blocks.

Table 2: Finite non-convex blocks in the edge of the tunnel

\begin{tabular}{ll}
\hline No. & Finite non-convex blocks in the edge of the tunnel \\
\hline 1 & $(00001,00010,00011,00012,00021)$ \\
2 & $(11100,11101,11102,11110,11120)$ \\
\hline
\end{tabular}

\section{Optimized Combination of Non-convex Block}

It is known that a non-convex block in the edge of the tunnel is composed of five finite convex blocks 11100, 11101, 11102, 11110, 11120. These five convex blocks are overlapped with each other. The further optimized combination can be represented with two convex blocks 11102 and 11110. The shape of the non-convex block can be determined, if all the convex blocks are drawn, shown as in Fig. 5.

\section{Analysis by Stereographic Projection}

The block system in the edge of the tunnel can be analyzed according to the stereographic projection. While the free planes are intersected concavely, the union operation should be adopted for the solid half-spaces. As for the joints intersected convexly, the operation is intersection. Let the direction of projection is $(0,0,1)$, the stereographic projection is shown in Fig. 6.

If gravity is considered only, let the vector of resultant is $(0.000,0.000,-1.000)$, the mode, the direction, the net force of sliding and the factor of safety can be obtained, as shown in Table 3.

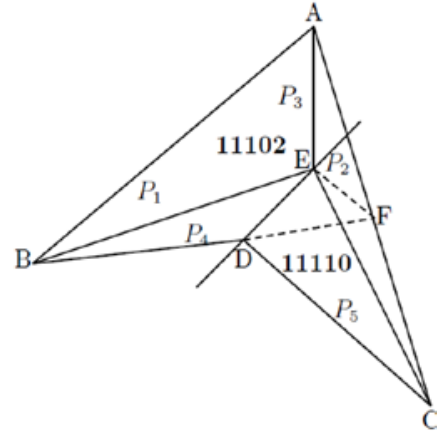

Fig.5: The non-convex block on the edge of the tunnel

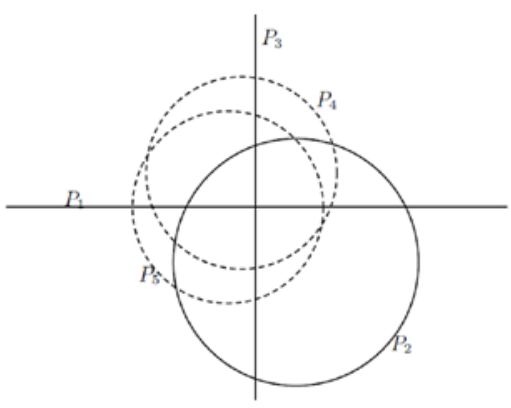

Fig.6: Stereographic projection analysis method

Table 3: The sliding modes, directions, net forces and factors of safety for the joint pyramids

\begin{tabular}{cccccc}
\hline No. & JP & modes & directions & net forces & FOS \\
\hline 1 & 000 & -1 & -- & +0.000000 & 0.000000 \\
2 & 010 & 0 & $(0.000,0.000,-1.000)$ & +1.000000 & 0.000000 \\
3 & 110 & 1 & $(-0.000,-0.409,-0.913)$ & -0.999899 & 0.000101 \\
4 & 101 & 2 & $(0.422,-0.422,-0.803)$ & +0.377558 & 0.466079 \\
5 & 011 & 3 & $(0.409,0.000,-0.913)$ & +0.999878 & 0.000122 \\
6 & 001 & 12 & $(0.482,-0.358,-0.800)$ & -0.134274 & 1.232283 \\
7 & 111 & 13 & $(0.378,-0.378,-0.845)$ & +0.999777 & 0.000223 \\
8 & 100 & 23 & $(0.358,-0.482,-0.800)$ & -0.192192 & 1.332550 \\
\hline
\end{tabular}

\section{Conclusions}

Concave edges can be formed by the intersection of roof and wall, floor and wall, wall and wall inside a tunnel. If the joints cross over the wall and roof or floor at the same time, an instable nonconvex block may be generated. In accordance with the identification criteria of finiteness and removability of united blocks, the analysis of enumeration is conducted at first on the system of 
spatial planes. All the finite convex blocks and removable ones can be searched out. Then all the possible non-convex blocks can be combined on the basis of the principles that the codes of the joint pyramids keep the same all the time. As for a determined non-convex block, cutting along the free planes, it can be represented with an optimized group of convex blocks which are connected only at the cutting planes. The example shows that the method is effective and correct, and will provides a valuable references on other complex combinations of free planes in a tunnel.

\section{Acknowledgements}

The study is supported by the Distinguished Research Project of North China University of Water Resources and Electric Power (40274) and the project of the National Natural Science Foundation of China (41402269, 51409102).

\section{References}

[1] SHI Genhua, A geometric method for stability analysis of discontinuous rocks, Scientia Sinica, 4, pp. 487-495, 1981.

[2] GOODMAN R E, SHI Genhua, Block theory and its application to rock engineering, New Jersey: Prentice-Hall, 1985.

[3] LI Jian-yong, XIAO Jun \& WANG Ying, Simulation method of rock stability analysis based on block theory. Computer Engineering and Applications, 46(21), pp. 4-8, 2010.

[4] ZHANG Zixin, SUN Jun, Fractal block theory and its application to the stability analysis of high slope in the Three Gorges project of Yangtze River. Journal of Natural Disasters, 4(4), pp. 89-95, 1995.

[5] HUANG Zhengjia, WU Aiqing \& SHENG Qian, Usage of block theory in the Three Gorges Projects. Chinese Journal of Rock Mechanics and Engineering, 20(5), pp. 648-652, 2001.

[6] A. R. Yarahmadi Bafghi, T. Verdel, The key-group method. International Journal for Numerical and Analytical Methods in Geomechanics, 27, pp. 495-511, 2003.

[7] WU Ai-qing, ZHANG Qi-hua, Geometric identification of stochastic block in block theory. Journal of Hydraulic Engineering, 36(4), pp.426-432, 2005.

[8] YU Qing-chun, CHEN De-ji, XUE Guo-fu, et al., Preliminary study on general block method of fractured rock mass. Hydrogeology and Engineering Geology, 6, pp. 42-48, 2005.

[9] SHI Genhua, Producing joint polygons, cutting joint blocks and finding key blocks for general free surfaces. Chinese Journal of Rock Mechanics and Engineering, 25(11), pp. 2161-2170, 2006.

[10]ZHANG Qihua, WU Aiqing, General methodology of spatial block topological identification with stochastic discontinuities cutting. Chinese Journal of Rock Mechanics and Engineering, 26(10), pp. 2043-2048, 2007. 